\title{
Effects of visual experience on the human MT+ functional connectivity networks: an fMRI study of motion perception in sighted and congenitally blind individuals
}

\author{
Lorenzo Sani ${ }^{1,2}$, Emiliano Ricciardi ${ }^{1,3}$, Claudio Gentili, ${ }^{3,4}$, Nicola Vanello ${ }^{3,5}$, James V. Haxby ${ }^{6}$ and Pietro Pietrini ${ }^{1,2 *}$ \\ 1 Laboratory of Clinical Biochemistry and Molecular Biology, University of Pisa, Pisa, Italy \\ 2 Department of Laboratory Medicine and Molecular Diagnostics, Azienda Ospedaliero Universitaria Pisana, Pisa, Italy \\ ${ }^{3}$ MRI Laboratory, Fondazione CNR/Regione Toscana Gabriele Monasterio, Pisa, Italy \\ ${ }^{4}$ Clinical Psychology Unit, Department of Psychiatry, University of Pisa, Pisa, Italy \\ ${ }^{5}$ Department of Information Engineering, University of Pisa, Pisa, Italy \\ ${ }^{6}$ Department of Psychological and Brain Sciences, Dartmouth College, Hanover, VA, USA
}

\section{Edited by:}

Silvina G. Horovitz, National Institutes of Health, USA

Reviewed by:

Victor Hugo de Lafuente Flores, University of Washington School of Medicine, USA

George Wittenberg, University of

Maryland School of Medicine, USA

*Correspondence:

Pietro Pietrini, Department of Laboratory Medicine and Molecular Diagnostics, University of Pisa Medical School, Via Roma, 67 I-56126 Pisa, Italy.

e-mail: pietro.pietrini@med.unipi.it
Human middle temporal complex (hMT+) responds also to the perception of non-visual motion in both sighted and early blind individuals, indicating a supramodal organization. Visual experience, however, leads to a segregation of hMT+ into a more anterior subregion, involved in the supramodal representation of motion, and a posterior subregion that processes visual motion only. In contrast, in congenitally blind subjects tactile motion activates the full extent of hMT+. Here, we used fMRI to investigate brain areas functionally connected with the two hMT+ subregions (seeds) during visual and tactile motion in sighted and blind individuals. A common functional connectivity network for motion processing, including bilateral ventral and dorsal extrastriate, inferior frontal, middle and inferior temporal areas, correlated with the two hMT+ seeds both in sighted and blind individuals during either visual or tactile motion, independently from the sensory modality through which the information was acquired. Moreover, ventral premotor, somatosensory, and posterior parietal areas correlated only with the anterior but not with the posterior portion of hMT+ in sighted subjects, and with both hMT+ seeds in blind subjects. Furthermore, a correlation between middle temporal and occipital areas with primary somatosensory seeds was demonstrated across conditions in both sighted and blind individuals, suggesting a cortico-cortical pathway that conveys non-visual information from somatosensory cortex, through posterior parietal regions, to ventral extrastriate cortex. These findings expand our knowledge about the development of the functional organization within hMT+ by showing that distinct patterns of brain functional correlations originate from the anterior and posterior $\mathrm{hMT}+$ subregions as a result of visual experience.

Keywords: human middle temporal complex, supramodality, visual motion, tactile motion, blindness, functional connectivity, fMRI

\section{INTRODUCTION}

Visual perception of motion in humans activates specific areas of the temporo-occipital cortex that classically includes the human middle temporal complex, hMT+ (Watson et al., 1993; Zeki et al., 1993; Tootell et al., 1995). This extrastriate area is activated also during apparent and illusory visual motion, and mental imagery of movement (Tootell et al., 1995; Goebel et al., 1998; Kourtzi and Kanwisher, 2000; Mather et al., 2008). Moreover, hMT+ responds to the perception of auditory and tactile motion in sighted (Hagen et al., 2002; Ricciardi et al., 2007; Ptito et al., 2009; Summers et al., 2009), as well as in congenitally blind individuals (Poirier et al., 2006; Ricciardi et al., 2007). These latter findings indicate that hMT+ also processes non-visual sensory inputs of motion, and that visual experience is not a prerequisite for the development of the functional organization of this motion-responsive area. In addition, these results extend to motion-responsive temporo-occipital areas the supramodal functional organization that has been previously demonstrated for other "visual" ventral and dorsal extrastriate cortical areas (Amedi et al., 2001, 2005; Pietrini et al., 2004; Ricciardi et al., 2006, 2009; Bonino et al., 2008).

Visual experience, however, does lead to a functional segregation within hMT+ (Ricciardi et al., 2007). Indeed, tactile motion perception in sighted subjects activated the more anterior portion of visual motion-responsive regions but deactivated the more posterior subregion that was activated by visual motion only. By contrast, perception of tactile motion in congenitally blind subjects activated the full extent of hMT+, including the more posterior part. In line with these findings, Beauchamp et al. (2007) using fMRI to localize functional responses to visual and tactile stimuli within $\mathrm{hMT}+$ demonstrated that the anterior and dorsal middle-superior temporal area (MST), but not the remaining portion, responded to simple vibrotactile stimuli.

Recently, repetitive transcranial magnetic stimulation (rTMS) was used to determine whether this more anterior portion of $\mathrm{hMT}+$ truly plays a functional role in tactile motion processing. 
In blindfolded sighted individuals who were asked to detect tactilely changes in the velocity of a random Braille-like dot pattern with the tip of their index and middle fingers, accuracy, and reaction times were significantly impaired only when rTMS was applied on the more anterior part of hMT+, but not on a control parietooccipital area (Ricciardi et al., 2010). These results indicate that $\mathrm{hMT}+$ recruitment is not an epiphenomenon but is truly necessary for tactile processing of motion, and provide additional evidence to the hypothesis of a "supramodal" functional organization for the more anterior part of this sensory motion processing area.

Over the last several years, increasing attention has been devoted to exploring interregional connectivity during a specific task, defined as "functional connectivity" (Friston et al., 1993; Horwitz, 2003; Horwitz et al., 2005). To characterize neural interactions across the whole brain, functional connectivity analyses select a voxel, or a group of voxels, as a reference (the so-called seed region of interest-seed-ROI), and cross-correlate the seed-ROI time course with the whole brain to identify functionally connected sites, i.e., brain regions showing high temporal coherence during a specific task or condition.

The present study was designed to examine the functional correlations between the more anterior "supramodal" and the posterior "visual" portions of hMT+ and the whole brain during visual and tactile processing of motion and the effects of visual experience and, conversely, of the lack of visual experience, on the development of these correlations. Based on our previous findings discussed above (Ricciardi et al., 2007), we hypothesized that the more anterior "supramodal" portion of hMT+ would show correlations not only within visual occipital areas but also with non-visual sensory brain area, or areas of visuo-tactile integration, such as sensorimotor and posterior parietal regions (Bremmer et al., 2001; Grefkes et al., 2002), independently from the specific motion detection task and from the occurrence of any visual experience. In contrast, we postulated that in sighted subjects the posterior part of hMT+ would show a wide pattern of correlation only within visual occipital areas. Finally, because perception of tactile motion in congenitally blind subjects engaged the full extent of hMT+, we predicted that the absence of visual experience would lead posterior hMT+ subregion to develop a pattern of functional connectivity similar to that shown by the more anterior hMT+ portion.

\section{MATERIALS AND METHODS SUBJECTS}

For this study, we analyzed brain functional data obtained in a previously reported fMRI experiment (Ricciardi et al., 2007). In brief, we studied seven sighted (one female, $27 \pm 2$ years) and four blind (one female, $37 \pm 14$ years) right-handed healthy volunteers. Three subjects were blind from birth, and one became blind at the age of 2 years and had no recollection of any visual experience (causes of blindness: two congenital glaucoma, retinopathy of prematurity, and congenital optic atrophy). All subjects received medical, neurological and psychiatric examinations, and laboratory testing, including a structural brain MRI scan exam, to rule out history or presence of any disorder (other than blindness in the congenitally blind group) that could affect brain function and development. No subject was taking any psychotropic medication. All participants gave their written informed consent after the study procedures and potential risks had been explained. The study was conducted under a protocol approved by the Ethical Committee at the University of Pisa Medical School (Protocol n. 020850). All participants retained the right to withdraw from the study at any moment.

\section{IMAGE ACQUISITION}

fMRI images were acquired using a gradient echo echoplanar (GREEPI) sequence with a GE Signa 1.5 Tesla scanner (General Electric, Milwaukee, WI, USA). A scan cycle (repetition time, TR $=3000 \mathrm{~ms}$ ) was composed of 22-26 contiguous axial slices [5 mm thickness, field of view $(\mathrm{FOV})=24 \mathrm{~cm}$, echo time $(\mathrm{TE})=40 \mathrm{~ms}$, flip angle $=90^{\circ}$, image in plane resolution $=64 \times 64$ pixels $]$, and voxels dimensions were $3.75 \mathrm{~mm} \times 3.75 \mathrm{~mm} \times 5 \mathrm{~mm}$. We obtained 3-7 time series of 79 brain volumes (237 s) in all subjects while they perceived tactile motion stimuli and, in sighted subjects only, also 2-4 time series while they perceived visual motion stimuli.

High-resolution T1-weighted spoiled gradient recall (SPGR) images were obtained for each subject to provide detailed brain anatomy during structural image acquisition.

\section{EXPERIMENTAL TASK AND STIMULI}

Tactile stimuli were administered using an MR compatible device (Ricciardi et al., 2007) on a polystyrene table placed over the subjects' legs. Subjects' hands lay on the table with the index and middle fingers touching a plastic surface with dot patterns at the same time. Tactile stimuli were moving or static Braille-like dot (average $\varnothing: 1-1.5 \mathrm{~mm}$; height: $0.5-1 \mathrm{~mm}$ ) random patterns presented on a plastic flat surface (a $30-\mathrm{mm}$ wide band). Horizontal translation (left-to-right and right-to-left; density $1 \mathrm{dot} / \mathrm{cm}^{2}$, average distance: $9 \mathrm{~mm}$; speed: $2.2 \mathrm{~cm} / \mathrm{s}$ ) and rotation (clockwise and counterclockwise; density $2 \mathrm{dot} / \mathrm{cm}^{2}$, average distance: $6 \mathrm{~mm}$; speed: $93.5 \% \mathrm{~s}$ ) motion were used. For the visual tasks, participants were asked to fixate a central static white cross $\left(0.15^{\circ} \times 0.15^{\circ}\right)$ while moving or static white dots were presented on a black background (dot radius: $0.06^{\circ}$, luminance about $20 \mathrm{~cd} / \mathrm{m}^{2}$ ). The same two types of motion as for the tactile motion task were used in the visual task: horizontal translation $\left(1.8^{\circ} \%\right)$ and rotation $\left(9^{\circ} / \mathrm{s}\right)$. Visual stimuli were presented on a rear projection screen viewed through a mirror (visual field: $25^{\circ}$ wide and $20^{\circ}$ high).

Moving stimuli were presented in 8-40 s blocks separated by intervals with static stimuli of varying duration (11 $\pm 10 \mathrm{~s})$. Each time series began and ended with 30 s of static stimuli. Participants were asked to fixate the central cross during the visual task, and to keep their eyes closed during the tactile tasks. Sensory modality (tactile or visual), type of movement, direction of movement, and hand of stimulation (left or right) were constant for each time series, and were presented in a pseudo-random sequence counterbalanced within and across subjects.

\section{DATA ANALYSIS}

The AFNI and SUMA software package was used to analyze functional imaging data (http://afni.nimh.nih.gov/afni, Cox, 1996). Based on the local maxima of group $Z$ maps in sighted subjects during the tactile motion perception task (Ricciardi et al., 2007), the ROIs to be used as the seeds for the whole brain functional connectivity correlation analysis in tactile runs were identified bilaterally in a more anterior (aMT in Figure 1; Talairach atlas coordinates: $X=44, Y=-48, Z=-5$; 


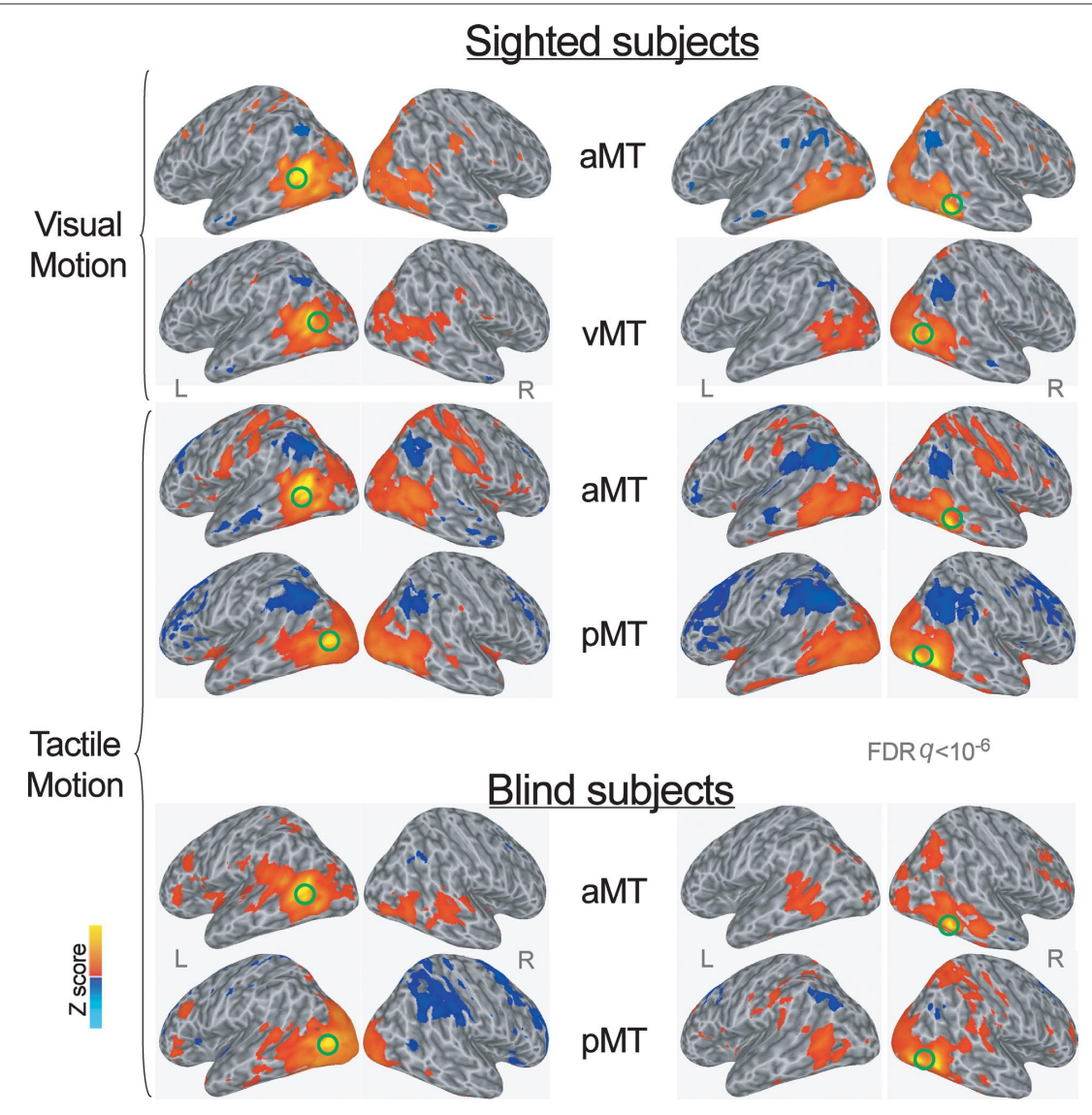

FIGURE 1 | Group averaged $Z$ map showing brain regions significantly correlated with the average time series extracted from the left and right more anterior and more posterior subregions of the hMT+ during visual and tactile motion perception in sighted subjects, and during tactile motion perception in blind subjects (FDR corrected $q<10^{-6}$ ).
Spatially normalized activations are projected onto a single-subject cortical surface template in the Talairach-Tournoux standard space. Lateral views of the inflated right and left hemispheres are shown. Green circles identify the seed-ROIs used for the functional connectivity correlation analysis.
$X=-49, Y=-62, Z=5$ ) and a more posterior (pMT in Figure 1; Talairach atlas coordinates: $X=44, Y=-70, Z=-4 ; X=-46, Y=-77$, $Z=2$ ) subregion of hMT+ (Figure 1). Tactile motion perception task in sighted subjects activated the more anterior portion of hMT+, but deactivated the more posterior subregion that, in contrast, was activated by visual motion only (Ricciardi et al., 2007).

Similarly, based on the local maxima of group $Z$ maps in sighted subjects during the visual motion perception task (Ricciardi et al., 2007), the local maxima of hMT+ (vMT in Figure 1; Talairach atlas coordinates: $X=43, Y=-73, Z=7 ; X=-46, Y=-62, Z=7)$ was used as the seed-ROI in visual runs. This visual seed-ROI almost overlapped with the more posterior subregion of hMT+ (pMT) as defined during the tactile motion task. The anterior hMT+ subregion (aMT) was used as a seed-ROI for the whole brain functional connectivity correlation analysis also for the visual runs.

Consistently, since blind individuals activated the whole extent of hMT+ during tactile motion perception (Ricciardi et al., 2007), for the functional connectivity analysis we located two seed-ROIs on the same coordinates for the anterior (aMT) and posterior (pMT) subregions of hMT+, as defined during the tactile motion perception task in the sighted subjects (see above).
Finally, to explore the hypothesis of a cortico-cortical pathway that from primary somatosensory cortex, through posterior parietal regions, would convey non-visual (tactile, in this case) information to the supramodal extrastriate cortex (Kupers et al., 2006; Peltier et al., 2007; Fujii et al., 2009; Matteau et al., 2010), we selected seed-ROIs also within the primary somatosensory (S1) regions (Talairach atlas coordinates- sighted, tactile runs: $X=44$, $Y=-29, Z=49, X=-43, Y=-28, Z=54$; sighted, visual runs: $X=51, Y=-29, Z=44, X=-51, Y=-20, Z=46$; blind, tactile runs: tactile runs: $X=47, Y=-24, Z=47, X=-42, Y=-37$, $Z=60)$. Definition of the seed-ROI in somatosensory cortex (S1) was based on the local maxima for each condition group $Z$ maps for sighted and blind individuals, respectively (Ricciardi et al., 2007). As explained below, significant voxels $(p<0.0005$; $Z$-score $>3.48$ ) falling within $5 \mathrm{~mm}$-radius spheres centered at local peak values for the selected ROIs were used to extract individual averaged time series. All raw volumes from the different runs were concatenated and coregistered, temporally aligned, and spatially smoothed (isotropic Gaussian filter, $\sigma=3.4 \mathrm{~mm}$ ). Individual runs data were normalized by calculating the mean intensity value for each voxel, and by dividing the value within 
each voxel by its mean to estimate the percent signal change at each time point. Effects of no interest (baseline shifts and linear/ quadratic drifts) were removed from the pre-processed EPI time series of each individual run, and a low-pass filtering (filter cut-off frequency $=0.1 \mathrm{~Hz}$ ) was performed on the signal time series of each voxel to eliminate high-frequency noises (Fox et al., 2005; Liang et al., 2006). Individual time series were then spatially transformed into the standard Talairach and Tournoux Atlas (Talairach and Tournoux, 1988) coordinate system, resampled to $1 \mathrm{~mm}^{3}$ voxels, and averaged across all voxels of a seed-ROI to derive a reference signal time course for each visual or tactile run. The extracted time series were used as regressors of interest in a whole brain multiple regression analysis. For each subject, the global signal (averaged signal across whole brain voxels) and the six movement parameters derived from the volume spatial registration in each scan series were included in the correlation analysis as covariates (i.e., regressors of no interest), in order to factor out signal changes due respectively to physiological noise (e.g., cardiac or respiratory artifacts; Cordes et al., 2001; Birn et al., 2006) or head movements (Jiang et al., 1995; Lowe et al., 1998). The task-related regressor (tactile or visual moving stimuli) of each run was also included as an additional regressor of no interest in the correlation analysis in order to minimize the effect of the specific tasks (Whalley et al., 2005). For each subject, output maps of the correlation coefficients represented the functional connectivity maps of the considered seed-ROI with the whole brain. In order to run group analyses, to reduce skewness and improve the normality of the distribution of the correlation coefficients, correlation coefficients were converted to $Z$-scores using Fisher's $Z$ transformation formula. For each ROI, individual and group $Z$-score correlation coefficient maps were computed by multiplying in two different steps the across runs and across subjects average $Z$ correlation coefficient values by the square root of the number of runs, respectively. Significant correlations were defined by a false discovery rate (FDR) corrected $q<10^{-6}$. Conjunction maps were created by overlapping significant correlations maps for each group and each task condition, in order to improve the description of the common network for motion processing functionally connected with both subregions of hMT+, and the distinctive patterns of functional correlations of the more anterior and posterior subregions of hMT+.

\section{RESULTS \\ FUNCTIONAL CONNECTIVITY OF ANTERIOR AND POSTERIOR hMT+ SUBREGIONS IN SIGHTED INDIVIDUALS}

During the visual motion perception task in sighted subjects, both the anterior "supramodal" and posterior "visual" seed-ROIs within hMT+ (respectively, aMT and vMT in Figure 1; yellow clusters in Figure 2, top row) showed correlations bilaterally, with a larger ipsilateral extension, with ventral extrastriate areas, including middle temporal, middle occipital and fusiform regions, and dorsal-occipital cortex. However, while the more anterior "supramodal" seed-ROI within hMT+ correlated bilaterally also with precentral and postcentral cortex, inferior and superior parietal areas, intraparietal cortex, dorsal premotor (BA6), dorsal (BA9) and anterior (BA46) middle frontal regions (aMT in Figure 1; red clusters in Figure 2, top row), the posterior "visual" hMT+ subregion showed additional

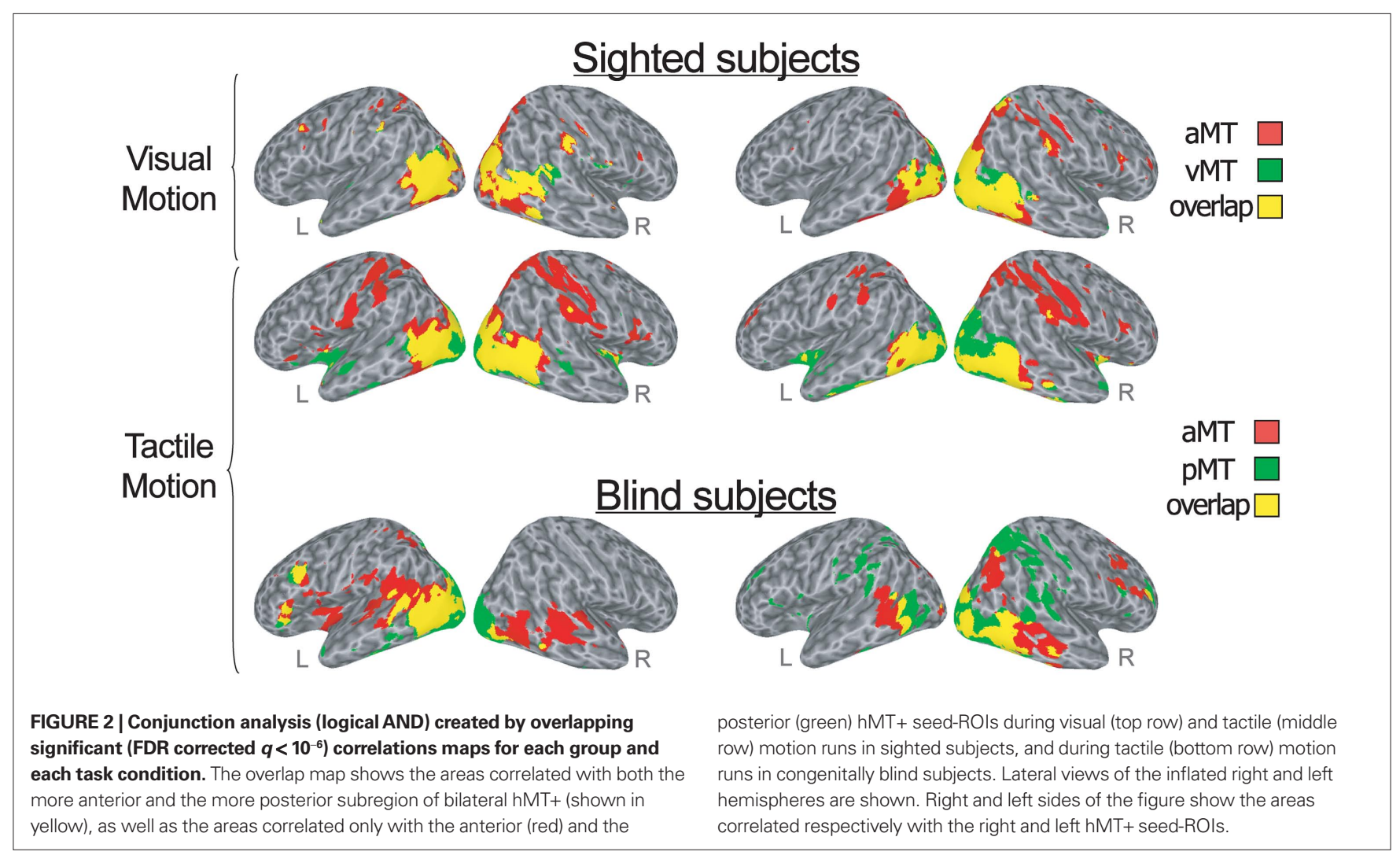


correlations only with ipsilateral precuneus and middle prefrontal cortex, and with small clusters in bilateral temporo-parietal cortex (vMT in Figure 1; green clusters in Figure 2, top row).

During the tactile motion perception runs in sighted subjects, both the anterior and posterior subregions of left and right hMT+ showed positive correlations extensively in bilateral striate and in ventral and dorsal extrastriate regions, including fusiform, parahippocampal and lingual gyri, middle and inferior temporal areas (aMT and pMT in Figure 1; yellow clusters in Figure 2, mid row). Clusters of correlations for both hMT+ seed-ROIs were also found in inferior frontal/insular areas and subcortical structures, such as the thalamus and putamen. As also was the case during visual motion perception, while the more anterior "supramodal" seedROIs significantly correlated with bilateral precentral and postcentral cortex, inferior and superior parietal areas, intraparietal cortex, dorsal premotor (BA6), middle (BA9) and inferior frontal (BA44) regions (aMT in Figure 1; red clusters in Figure 2, mid row), the posterior "visual" subregion of hMT+ showed additional positive correlations only with the cuneus and anterior temporal regions (pMT in Figure 1; green clusters in Figure 2, mid row).

\section{FUNCTIONAL CONNECTIVITY OF ANTERIOR AND POSTERIOR hMT+ SUBREGIONS IN CONGENITALLY BLIND INDIVIDUALS}

In the congenitally blind group, both the anterior and posterior seed-ROIs showed similar patterns of functional correlations bilaterally, with a larger ipsilateral extension, in middle temporal and middle occipital cortex, dorsal occipital and superior parietal areas, postcentral somatosensory cortex, temporo-parietal, ventral premotor, and anterior middle frontal clusters (aMT and pMT in Figures 1 and 2, bottom row). While the anterior seed-ROIs of hMT+ showed a broader correlation with bilateral middle and superior temporal cortex and a more distributed connection with insular and anterior prefrontal areas (aMT in Figure 1; red clusters in Figure 2, bottom row), the posterior seed-ROIs of hMT+ presented a significant functional connection also with medial occipital areas, such as lingual gyri and cuneus (pMT in Figure 1; green clusters in Figure 2, bottom row).

\section{FUNCTIONAL CONNECTIVITY OF S1 DURING TACTILE AND VISUAL MOTION PERCEPTION}

During the tactile motion perception task in both sighted and congenitally blind individuals, the somatosensory seed-ROIs showed extensive bilateral connections with contiguous paracentral cortex, ventral and dorsal premotor areas, supplementary motor, posterior parietal and intraparietal regions, anterior middle, and superior frontal clusters (Figure 3). Furthermore, both the left and right S1 seed-ROIs were functionally connected with bilateral middle temporal and lateral occipital areas, thus including also supramodal ventral and motion-responsive extrastriate regions.

A similar pattern was also described for the somatosensory seedROIs during visual motion perception in sighted subjects, including the connection with motion-responsive extrastriate regions.

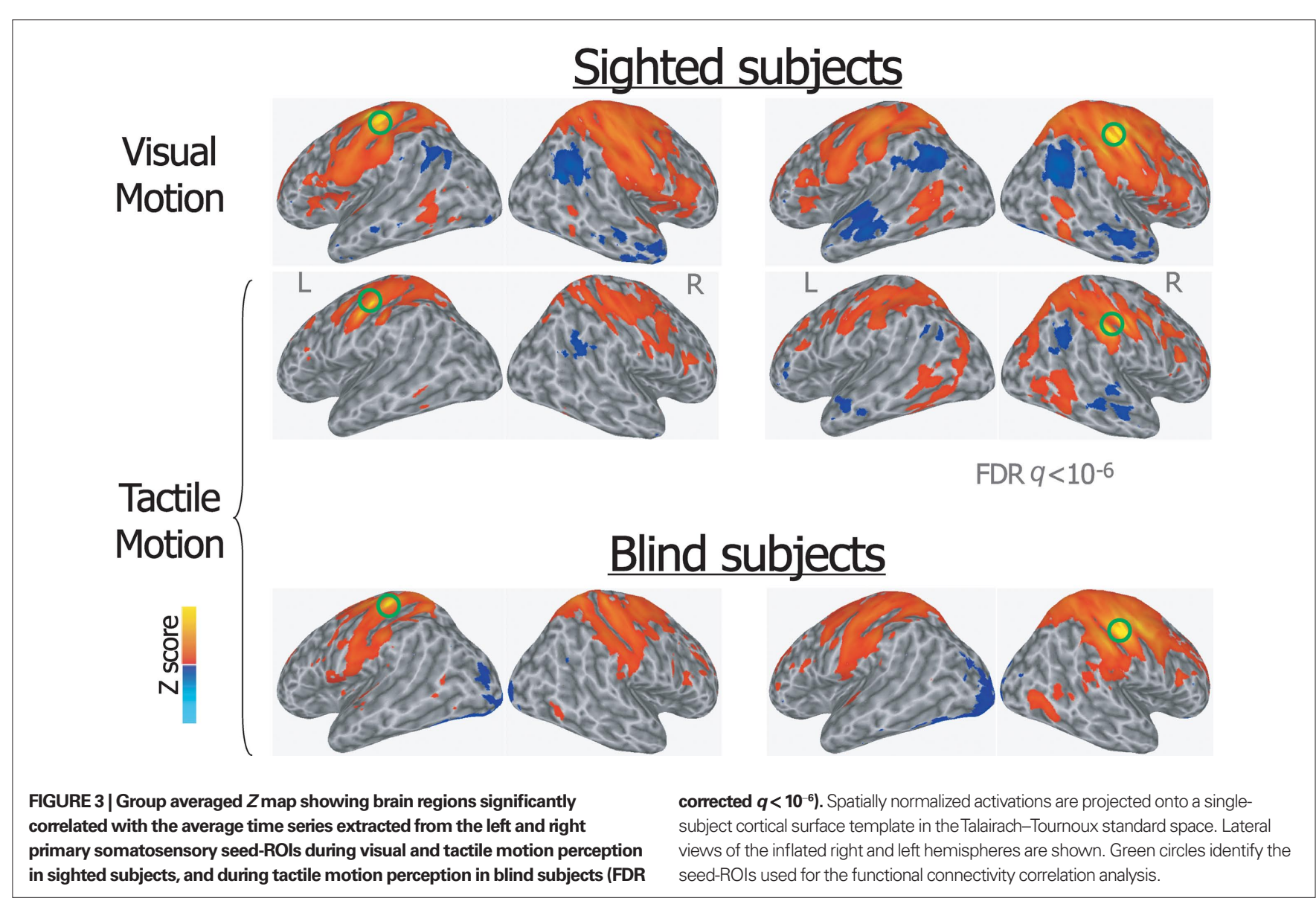




\section{DISTRIBUTION OF NEGATIVE FUNCTIONAL CONNECTIVITY CORRELATIONS COEFFICIENTS}

In addition to the positive correlations, significant negative correlations were found both in sighted subjects during visual and tactile motion perception and in blind subjects during tactile motion perception. Specifically, the bilateral seed-ROIs in both hMT+ and primary somatosensory cortex showed bilateral significant negative correlations with the temporoparietal junction, anterior cingulate and medial prefrontal areas, posterior cingulate, and precuneus (Figures 1 and 3).

\section{DISCUSSION}

The aim of the present study was to explore the functional correlations between hMT+ and the rest of the brain during visual and tactile motion processing tasks and the effects of visual experience and, conversely, of lack of visual experience, on the development of these correlations. Specifically, we wished to determine patterns of functional correlations between the more anterior "supramodal" and the posterior "visual" subregions of hMT+, respectively, and the other brain areas during visual and tactile perception of moving dot patterns in both sighted and congenitally blind individuals.

\section{A COMMON NETWORK FOR MOTION PROCESSING FUNCTIONALLY CONNECTED WITH BOTH SUBREGIONS OF hMT+}

Overall, we found that, both in sighted and congenitally blind subjects, a common set of bilateral brain areas, including ventral and dorsal extrastriate regions - such as the fusiform, parahippocampal and lingual cortex - middle and inferior temporal areas, and inferior frontal areas were positively correlated with both seed-ROIs in the anterior and posterior subregions of hMT+, during either the visual or tactile motion perception runs. This network is consistent with other brain functional studies that have reported hMT+ to be mutually activated along with many of these visual motion processing regions (Watson et al., 1993; Zeki et al., 1993; Tootell et al., 1995; Shulman et al., 1998; Hampson et al., 2004).

Therefore, these brain areas have developed a common network for motion processing that is functionally correlated with the whole $\mathrm{hMT}+$, does not depend from the sensory modality through which the information is acquired and does not require visual experience to form (Poirier et al., 2006; Ricciardi et al., 2007, 2010; Ptito et al., 2009). Interestingly, the observation that similar functional connectivity networks obtained using the hMT+ seed-ROIs are present also in congenitally blind subjects during tactile motion perception indicates that the involvement of hMT+ in non-visual motion detection cannot be interpreted merely as a consequence of visual imagery (Beauchamp et al., 2007; Ricciardi et al., 2007; Matteau et al., 2010). Altogether, these results are consistent with and extend to the hMT+ connectivity networks the supramodal functional organization that has been shown both in the ventral and dorsal "visual" cortical pathways in relation to object recognition and motion and spatial discrimination (Pietrini et al., 2004; Amedi et al., 2005; Ricciardi et al., 2006, 2007; Beauchamp et al., 2008; Cattaneo et al., 2008; Mahon et al., 2009; Matteau et al., 2010), and in other prefrontal and parietal cortical areas in relation to higher-order cognitive functions, such as mental imagery, working memory, and action recognition (Bonino et al., 2008; Cattaneo et al., 2008; Ricciardi et al., 2009). As a whole, the results of the above studies indicate that visual experience is not a mandatory prerequisite for the brain to develop its morphological and functional architecture (Pietrini et al., 2004, 2009).

\section{A NEGATIVE FUNCTIONAL CORRELATIONS}

In sighted subjects during visual and tactile motion perception and in blind subjects during tactile motion perception, the bilateral seed-ROIs of both hMT+ and primary sensorimotor cortex showed significant negative correlations in the bilateral temporoparietal junction, bilateral posterior cingulate and bilateral anterior cingulate. Interestingly, these brain regions belong to the so-called default mode network (Raichle et al., 2001; Greicius and Menon 2003). Since functional connectivity is a measure of the spatiotemporal synchrony, or correlation, of the fMRI signal between anatomically distinct brain regions within the cerebral cortex (Friston et al., 1993; Biswal et al., 1995), these negative correlations may likely indicate that the hMT+ and S1 seed-ROIs (that are "task responsive") are significantly anti-correlated with the default mode network regions (that are "rest responsive"). Even if negative correlations may be sometimes an artifact of global signal regression techniques, including those applied here (Chang and Glover, 2009; Murphy et al., 2009), these findings reflect the expected anticorrelation with brain regions that commonly exhibit activity decreases during the performance of various goal-directed tasks, and that have been previously reported as anticorrelated to brain regions routinely activated during goaldirected task performance (Raichle et al., 2001; Greicius and Menon, 2004; Fox et al., 2005). The neurophysiological meaning of fMRI negative correlations, which remains yet to be fully understood, falls much outside of the specific aim of this study.

\section{DISTINCTIVE PATTERNS OF FUNCTIONAL CORRELATIONS OF THE MORE ANTERIOR AND POSTERIOR SUBREGIONS OF hMT+}

In addition to this common network of brain regions for motion processing, however, differences between the patterns of functional correlations for the anterior and posterior hMT+ subregions were identified in the two groups.

In the sighted subjects, the functional connectivity network obtained using the bilateral anterior "supramodal" seeds of hMT+ differed from the set of brain regions connected to the posterior "visual" seed of this motion-responsive area during both visual and tactile motion perception. As hypothesized, in sighted subjects the posterior part of hMT+ showed a wider pattern of correlation within visual occipital areas, while the more anterior portion of $\mathrm{hMT}+$ revealed correlations also with non-visual sensory brain area, or areas of visuo-tactile integration, such as somatosensory and posterior parietal cortex, independently from the specific motion detection task. Specifically, premotor and somatosensory regions, inferior and superior parietal areas, intraparietal, dorsal premotor (BA6), middle (BA9), and inferior frontal (BA44) cortex were functionally correlated with the anterior "supramodal" subregion of hMT+, but not with the posterior "visual" one.

In the congenitally blind subjects, during the tactile motion perception tasks both the anterior and the posterior part of bilateral $\mathrm{hMT}+$ revealed a pattern of functional correlations with the anterior middle frontal, ventral premotor, somatosensory and posterior parietal areas, that was similar to the network identified using the anterior "supramodal" subregion in the sighted subjects. 
Thus, these findings show that the anterior subregion of the motion-responsive occipito-temporal cortex develops functional correlation networks that do not depend on visual experience and that are equally elicited by both visual and tactile motion processing, extending previous findings on the supramodal nature of this cortical region to its connectivity networks (Poirier et al., 2006; Beauchamp et al., 2007; Ricciardi et al., 2007; Summers et al., 2009; Matteau et al., 2010). In contrast, the posterior subregion, that in sighted subjects processes visual motion only, develops patterns of functional regional correlations across the brain that differ between sighted and congenitally blind individuals. Moreover, the similarity between the anterior and posterior hMT+ subregionrelated functional networks in congenitally blind individuals suggests that in the absence of visual experience the functional development of the more "visual" motion-responsive region and its related functional connectivity proceed toward the representation of non-visual motion (Wittenberg et al., 2004; Ricciardi et al., 2007; Fujii et al., 2009). As a result, in people lacking of visual experience since birth, the posterior "visual" part of hMT+ that in sighted individuals correlates more with related visual occipital areas, extends its functional connections also to areas of multisensory integration, such as somatosensory and posterior parietal cortex.

\section{A CORTICO-CORTICAL PATHWAY MAY SUBSERVE SUPRAMODAL RESPONSES IN THE VISUAL CORTEX}

Which mechanisms subserve these supramodal responses in the "visual" motion sensitive cortex of both sighted and congenitally blind individuals? Which neural pathways mediate the functional correlation between extrastriate motion-responsive regions and somatosensory and posterior parietal cortex both in sighted and blind individuals? Interestingly, in both experimental groups, when considering S1-seeded functional networks, somatosensory areas showed extensive bilateral connections with contiguous posterior parietal and intraparietal regions, and with middle temporal and lateral occipital areas. Similarly, the anterior supramodal subregion of hMT+ in sighted individuals, and both the anterior and posterior portions of hMT+ in congenitally blind subjects, showed a functional correlation with ventral premotor, sensorimotor, and posterior parietal areas. Thus, our data support a cortico-cortical pathway from primary somatosensory cortex through posterior parietal regions to the supramodal extrastriate areas, in line also with previous anatomical and functional studies (Kupers et al., 2006; Peltier et al., 2007; Fujii et al., 2009; Matteau et al., 2010). A concomitant involvement of a subcortical loop between the two sensory cortical areas also has been proposed (Cowey, 2010). In this respect, a functional and effective connectivity fMRI study recently indicated a direct functional connection between the thalamus and hMT+, that would enable motion information to reach hMT+ directly from the thalamus bypassing V1 (Gaglianese et al., 2010).

These anatomical connections, viable also in physiological conditions as demonstrated in the brain of blindfolded sighted subjects who perform object, spatial, and motion discrimination tasks through the tactile or auditory modality (Pietrini et al., 2004, 2009; Amedi et al., 2005; Ricciardi et al., 2006; Bonino et al.,
2008), may then undergo a cross-modal plastic reorganization and become more robust in those individuals who lose sight at birth or in the early post-natal period (Wittenberg et al., 2004; Ptito and Kupers, 2005; Kupers et al., 2006, 2010; Ptito et al., 2008; Pietrini et al., 2009).

\section{LIMITATIONS OF THE STUDY}

The present study of brain regional functional correlations suffers from some methodological limitations. In the first place, the number of subjects recruited into the study is relatively small, especially for the blind group. While this issue may limit the generability of the findings, it should be kept in mind that congenitally blind individuals represent an exceptionally rare population, and even more so when strict medical selection criteria are adopted as in this study. As a matter of facts, several recently published brain imaging studies on congenitally blind individuals examined a similar or even smaller sample of subjects (Mahon et al., 2009, 2010).

Another limitation of this study is the lack of functional localizers and the relatively low field strength to properly ensure that the seed-ROIs may not overlap, even in part, with neighbor supramodal areas in lateral occipital cortex (LOtv - Amedi et al., 2001), or superior temporal sulcus (multisensory STSms - Beauchamp et al., 2008). However, the localization of the anterior seed-ROIs of hMT+ is sufficiently distant from the more dorsal and anterior STSms center-of-mass $(x:-44, y:-35, z: 13)$ and the more ventral LOtv localization ( $x:-45 \pm 5, y:-62 \pm 6, z:-9 \pm 3)$.

Finally, here we evaluated functional correlations of the distinct $\mathrm{hMT}+$ subregions across different task conditions without having a resting-state condition, that would have been the optimal paradigm to assess the distinctive functional role of the two hMT+ subregions in the two experimental groups. However, modeling of the experimental paradigm in our analysis as a regressor of no interest contributes to mitigate the task-associated changes in BOLD responses (Whalley et al., 2005). The attempt to "subtract" task-related effects should enhance the specific functional features of the connectivity patterns in the two experimental groups, independently from taskrelated changes in regional activity, as indicated by the similarity of functional correlation maps during the different tasks in the sighted group. Indeed, the correlations between BOLD variations at rest have been found to reflect patterns of known connectivity in different cognitive operations (Hampson et al., 2004; Mennes et al., 2010).

In summary, these findings expand our previous results on the development of the functional organization of hMT+ by showing that distinct patterns of brain functional correlations originate from the anterior and posterior hMT+ subregions, and that these functional correlation patterns are differentially affected by visual experience. As a matter of facts, in congenitally blind subjects both the anterior and the posterior part of bilateral hMT+ revealed similar patterns of functional correlations, thus indicating that in the absence of visual experience brain regions responsive to visual motion develop toward processing of non-visual inputs. Along with previous studies by our and other laboratories, these findings provide a new perspective on how the human brain develops its functional organization in relation to the presence or absence of visual experience. 


\section{ACKNOWLEDGMENTS}

The authors wish to thank all the volunteers who took part in the study, and the Unione Italiana Ciechi for its invaluable support to this project; the MRI Laboratory at the Fondazione Regione Toscana/C.N.R. "G. Monasterio" (Pisa, Italy), coordinated by

\section{REFERENCES}

Amedi, A., Malach, R., Hendler, T., Peled, S., and Zohary, E. (2001).Visuo-haptic object-related activation in the ventral visual pathway. Nat. Neurosci. 4, 324-330.

Amedi, A., von Kriegstein, K., van Atteveldt, N. M., Beauchamp, M. S., and Naumer, M. J. (2005). Functional imaging of human crossmodal identification and object recognition. Exp. Brain Res. 166, 559-571.

Beauchamp, M. S., Yasar, N. E., Frye, R. E., and Ro, T. (2008). Touch, sound and vision in human superior temporal sulcus. Neuroimage 41, 1011-1020.

Beauchamp, M. S., Yasar, N.E., Kishan, N., and Ro, T. (2007). Human MST but not MT responds to tactile stimulation. J. Neurosci. 27, 8261-8267.

Birn, R. M., Diamond, J. B., Smith, M. A., and Bandettini, P.A.(2006). Separating respiratory-variation-related fluctuations from neuronal-activity-related fluctuations in fMRI. Neuroimage 31, 1536-1548.

Biswal, B., Yetkin, F. Z., Haughton, V. M., and Hyde, J. S. (1995). Functional connectivity in the motor cortex of resting human brain using echo-planar MRI. Magn. Reson. Med. 34, 537-541.

Bonino, D., Ricciardi, E., Sani, L., Gentili, C., Vanello, N., Guazzelli, M., Vecchi, T., and Pietrini, P. (2008). Tactile spatial working memory activates the dorsal extrastriate cortical pathway in congenitally blind individuals. Arch. Ital. Biol. 146, 133-146.

Bremmer, F., Schlack, A., Shah, N. J., Zafiris, O., Kubischik, M., Hoffmann, K., Zilles, K., and Fink, G. R. (2001). Polymodal motion processing in posterior parietal and premotor cortex: a human fMRI study strongly implies equivalencies between humans and monkeys. Neuron 29, 287-296.

Cattaneo, Z., Vecchi, T., Cornoldi, C., Mammarella, I., Bonino, D., Ricciardi, E., and Pietrini, P. (2008). Imagery and spatial processes in blindness and visual impairment. Neurosci. Biobehav. Rev. 32, 1346-1360.

Chang, C., and Glover, G. H. (2009). Effects of model-based physiological noise correction on default mode network anti-correlations and correlations. Neuroimage 47, 1448-1459.

Cordes, D., Haughton, V. M., Arfanakis, K., Carew, J. D., Turski, P.A., Moritz, C. H., Quigley, M. A., and Meyerand, M. E. (2001). Frequencies contributing to functional connectivity in the cerebral cortex in resting-state data. AJNR Am. J. Neuroradiol. 22, 1326-1333.

Cowey, A. (2010). The blindsight saga. Exp. Brain Res. 200, 3-24.

Cox, R. W. (1996). AFNI: software for analysis and visualization of functional magnetic resonance neuroimages. Comput. Biomed. Res. 29, 162-173.

Fox, M. D., Snyder, A. Z., Vincent, J. L. Corbetta, M., Van Essen, D. C., and Raichle, M. E. (2005). The human brain is intrinsically organized into dynamic, anticorrelated functional networks. Proc. Natl. Acad. Sci. U.S.A. 102, 9673-9678.

Friston, K. J., Frith, C. D., Liddle, P. F., and Frackowiak, R. S. J. (1993). Functional connectivity: the principal-component analysis of large (PET) data sets. $J$. Cereb. Blood Flow Metab. 13, 5-14.

Fujii, T., Tanabe, H.C., Kochiyama, T., and Sadato, N. (2009). An investigation connectivity in the blind by dynamic causal modeling of functional MRI data. Neurosci. Res. 65, 175-186.

Gaglianese, A., Sani, L., Roebroeck, A., Handjaras, G., Bernardi, G., Costagli, M., Ricciardi, E., and Pietrini, P. (2010). fMRI Functional and Effective Connectivity in Humans Supports a Direct Pathway from Thalamus to $h M T+$. Presented at the 16th Annual Meeting of the Organization for Human Brain Mapping, Barcelona.

Goebel, R., Khorram-Sefat, D., Muckli, L., Hacker, H., and Singer, W. (1998). The constructive nature of vision: direct evidence from functional magnetic resonance imaging studies of apparent motion and motion imagery. Eur. J. Neurosci. 10, 1563-1573.

Grefkes, C., Weiss, P.H.,Zilles, K., and Fink, G. R. (2002). Crossmodal processing of object features in human anterior intraparietal cortex: an fMRI study implies equivalencies between humans and monkeys. Neuron 35, 173-184.

Greicius, M. D., Krasnow, B., Reiss, A. L., and Menon, V. (2003). Functional connectivity in the resting brain: a network analysis of the default mode hypothesis. Proc. Natl. Acad. Sci. U.S.A. 100, 253-258.

Hagen, M. C., Franzen, O., McGlone, F., Essick, G., Dancer, C., and Pardo, J. V. (2002). Tactile motion activates the human middle temporal/V5 (MT/ V5) complex. Eur. J. Neurosci. 16, 957-964. of cross-modal plasticity of effective

Massimo Lombardi. We also thank Giacomo Handjaras for his advice on data analysis. This research was supported by the European Union (Grant IST-2001-38040 to Pietro Pietrini) and by the Italian Ministero dell'Istruzione, dell'Università e della Ricerca (Grant RBNE018ET9-003 and Grant 2004118414 to Pietro Pietrini).

Hampson, M., Olson, I. R., Leung, H. C., Skudlarski, P., and Gore, J. C. (2004). Changes in functional connectivity of human MT/V5 with visual motion input. Neuroreport 15, 1315-1319.

Horwitz, B. (2003). The elusive concept of brain connectivity. Neuroimage 19, 466-470.

Horwitz, B., Warner, B., Fitzer, J., Tagamets M. A., Husain, F. T., and Long, T. W (2005). Investigating the neural basis for functional and effective connectivity. Application to fMRI. Philos. Trans. R. Soc. Lond., B, Biol. Sci. 360 1093-1108.

Jiang, A., Kennedy, D. N., Baker, J. R. Weisskoff, R. M., Tootell, B.H., Woods, R. P., Benson, R. R., Kwong, K. K., Brady, T. J., Rosen, B. R., and Belliveau, J. W. (1995). Motion detection and correction in functional MR imaging. Hum. Brain Mapp. 3, 224-235.

Kourtzi, Z., and Kanwisher, N. (2000). Activation in human MT/MST by static images with implied motion. J. Cogn. Neurosci. 12, 48-55.

Kupers, R., Chebat, D. R., Madsen, K. H., Paulson, O. B., and Ptito, M. (2010) Neural correlates of virtual route recognition in congenital blindness. Proc. Natl. Acad. Sci. U.S.A. 107, 12716-12721.

Kupers, R., Fumal, A., Maertens de Noordhout, A., Gjedde, A., Schoenen, J., and Ptito, M. (2006). Transcrania magnetic stimulation of the visual cortex induces somatotopically organized qualia in blind subjects. Proc. Natl. Acad. Sci. U.S.A. 103, 13256-13260.

Liang, M., Zhou, Y., Jiang, T., Liu, Z. Tian, L., Liu, H., and Hao, Y. (2006). Widespread functional disconnectivity in schizophrenia with resting-state functional magnetic resonance imaging. Neuroreport 17, 209-213.

Lowe, M. J., Mock, B. J., and Sorenson, J. A. (1998). Functional connectivity in single and multislice echo planar imaging using resting state fluctuations. Neuroimage 7, 119-132.

Mahon, B.Z., Anzellotti, S., Schwarzbach, J., Zampini, M., and Caramazza, A. (2009). Category-specific organization in the human brain does not require visual experience. Neuron 63, 397-405.

Mahon, B. Z., Schwarzbach, J., and Caramazza, A. (2010). The representation of tools in left parietal cortex is independent of visual experience. Psychol. Sci. 21,764-771.
Mather, G., Pavan, A., Campana, G., and Casco, C. (2008). The motion aftereffect reloaded. Trends Cogn. Sci. 12, 481-487.

Matteau, I., Kupers, R., Ricciardi, E., Pietrini, P., and Ptito, M. (2010). Beyond visual, aural and haptic movement perception: hMT+ is activated by electrotactile motion stimulation of the tongue in sighted and in congenitally blind individuals. Brain Res. Bull. 82, 264-270

Mennes, M., Kelly, C., Zuo, X. N., Di Martino, A., Biswal, B. B., Castellanos, F.X., and Milham, M. P. (2010). Interindividual differences in resting-state functional connectivity predict taskinduced BOLD activity. Neuroimage 50, 1690-1701.

Murphy, K., Birn, R. M., Handwerker, D. A., Jones, T. B., and Bandettini, P. A. (2009). The impact of global signal regression on resting state correlations: are anti-correlated networks introduced? Neuroimage 44, 893-905.

Peltier, S., Stilla, R., Mariola, E., LaConte, S., Hu, X., and Sathian, K. (2007). Activity and effective connectivity of parietal and occipital cortical regions during haptic shape perception. Neuropsychologia 45, 476-483.

Pietrini, P., Furey, M. L., Ricciardi, E., Gobbini, M. I., Wu, W. H., Cohen, L., Guazzelli, M., and Haxby, J. V. (2004). Beyond sensory images: object-based representation in the human ventral pathway. Proc. Natl. Acad. Sci. U.S.A. 101, 5658-5663.

Pietrini, P., Ptito, M., and Kupers, R. (2009). "Blindness and consciousness: new light from the dark," in The Neurology of Consciousness, eds S. Laureys and G. Tononi (Amsterdam: Academic Press), 360-374.

Poirier, C., Collignon, O., Scheiber, C. Renier, L., Vanlierde, A., Tranduy, D., Veraart, C., and De Volder, A. G (2006). Auditory motion perception activates visual motion areas in early blind subjects. Neuroimage 31, 279-285.

Ptito, M., Fumal, A., de Noordhout, A. M., Schoenen, J., Gjedde, A., and Kupers, R. (2008). TMS of the occipital cortex induces tactile sensations in the fingers of blind Braille readers. Exp. Brain Res. 184, 193-200.

Ptito, M., and Kupers, R. (2005). Crossmodal plasticity in early blindness. $J$. Integr. Neurosci. 4, 479-488. 
Ptito, M., Matteau, I., Gjedde, A., and Kupers, R. (2009). Recruitment of the middle temporal area by tactile motion in congenital blindness. Neuroreport 20, 543-547.

Raichle, M. E., MacLeod, A. M., Snyder, A. Z., Powers, W. J., Gusnard, D. A., and Shulman, G.L. (2001). A default mode of brain function. Proc. Natl. Acad. Sci. U.S.A. 98, 676-682.

Ricciardi, E., Basso, D., Bonino, D., Sani, L., Guazzelli, M., Vecchi, T., Pietrini, P., and Miniussi, C. (2010). Functional inhibition of the human middle temporal cortex affects non-visual motion perception: a repetitive transcranial magnetic stimulation study during tactile speed discrimination. Exp. Biol. Med. (Maywood) doi: 10.1258/ ebm.2010.010230.

Ricciardi, E., Bonino, D., Gentili, C., Sani, L., Pietrini, P., and Vecchi, T. (2006). Neural correlates of spatial working memory in humans: a functional magnetic resonance imaging study comparing visual and tactile processes. Neuroscience 139, 339-349.

Ricciardi, E., Bonino, D., Sani, L., Vecchi, T., Guazzelli, M., Haxby, J. V., Fadiga,
L., and Pietrini, P. (2009). Do we really need vision? How blind people "see" the actions of others. J. Neurosci. 29, 9719-9724.

Ricciardi, E., Vanello, N., Sani, L., Gentili, C., Scilingo, E. P., Landini, L., Guazzelli, M., Bicchi, A., Haxby, J.V., and Pietrini, P. (2007). The effect of visual experience on the development of functional architecture in hMT+. Cereb. Cortex 17, 2933-2939.

Shulman, G. L., Schwarz, J., Miezin, F. M., and Petersen, S. E. (1998). Effect of motion contrast on human cortical responses to moving stimuli. J. Neurophysiol. 79, 2794-2803.

Summers, I. R., Francis, S. T., Bowtell, R. W., McGlone, F. P., and Clemence, M. (2009). A functional-magneticresonance-imaging investigation of cortical activation from moving vibrotactile stimuli on the fingertip. J. Acoust. Soc. Am. 125, 1033-1039.

Talairach, J., and Tournoux, P. (1988). Co-Planar Stereotaxic Atlas of the Human Brain. New York: Thieme Classics.

Tootell, R. B., Reppas, J. B., Dale, A. M., Look, R. B., Sereno, M. I., Malach, R.,
Brady, T. J., and Rosen, B. R. (1995) Visual motion aftereffect in human cortical area MT revealed by functional magnetic resonance imaging. Nature 375, 139-141.

Watson, J. D., Myers, R., Frackowiak, R. S., Hajnal, J. V., Woods, R. P., Mazziota, J. C., Shipp, S., and Zeki, S. (1993). Area V5 of the human brain: evidence from a combined study using positron emission tomography and magnetic resonance imaging. Cereb. Cortex 3, 79-94.

Whalley, H. C., Simonotto, E., Marshall, I., Owens, D. G., Goddard, N. H., Johnstone, E. C., and Lawrie, S. M. (2005). Functional disconnectivity in subjects at high genetic risk of schizophrenia. Brain 128, 2097-2108.

Wittenberg, G. F., Werhahn, K. J., Wassermann, E. M., Herscovitch, P., and Cohen, L. G. (2004). Functional connectivity between somatosensory and visual cortex in early blind humans. Eur. J. Neurosci. 20, 1923-1927.

Zeki, S., Watson, J. D., and Frackowiak, R. S. (1993). Going beyond the information given: the relation of illusory visual motion to brain activity. Proc. Biol. Sci. 252, 215-222.

Conflict of Interest Statement: The authors declare that the research was conducted in the absence of any commercial or financial relationships that could be construed as a potential conflict of interest.

Received: 21 July 2010; accepted: 07 December 2010; published online: 20 December 2010

Citation: Sani L, Ricciardi E, Gentili C, Vanello N, Haxby JV and Pietrini P (2010) Effects of visual experience on the human $M T+$ functional connectivity networks: an fMRI study of motion perception in sighted and congenitally blind individuals. Front. Syst. Neurosci. 4:159. doi: 10.3389/ fnsys.2010.00159

Copyright (C) 2010 Sani, Ricciardi, Gentili, Vanello, Haxby and Pietrini. This is an open-access article subject to an exclusive license agreement between the authors and the Frontiers Research Foundation, which permits unrestricted use, distribution, and reproduction in any medium, provided the original authors and source are credited. 